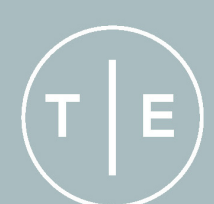

Trabajos de Egiptología

"Those Who Mourn for Re": Mourning and Regeneration in the Book of the Twelve Caverns

Daniel Miguel MÉNDEZ RODRíGUEZ

The Courtyard of TT 209 (Areas C1 and C2). Seasons 2012 to 2014

Miguel Ángel MOLINERO POLO, Cristo Manuel HERNÁNDEZ GÓMEZ.

Algunas reflexiones sobre Uluburun y el intercambio comercial y cultural en el Mediterráneo Oriental

$$
\text { Antonio PÉREZ LARGACHA }
$$

The Framework of the Meeting

Narrative Uses of Stelae in Egyptian Literary Texts José Ramón PÉREZ-ACCINO

Introducción a la investigación arqueológica para estudiantes a través de reconstrucciones virtuales

Sofía PÉREZ-RUIZ, Ainara ACEBO, Pilar RODRÍGUEZ MARíN...

Lucernas decoradas con la imagen de una rana del yacimiento de Oxirrinco, El-Bahnasa, Egipto Esther PONS MELLADO

\section{Trabajos de Egiptología}

\section{Papers on Ancient Egypt}

Estudio preliminar de la cerámica procedente de las excavaciones en la TT 209, Luxor, Egipto

$$
\text { Zulema BARAHONA MENDIETA }
$$

La ocupación macedónica y la Dinastía Lágida. Impacto político, económico y social Francisco BOSCH PUCHE

Reflexiones sobre Meretseguer en la estela EA272 del British Museum

Elisa CASTEL RONDA

Ramesses III as Guarantor of Maat: the Iconographic Evidence at Medinet Habu

$$
\text { Salvador COSTA, Teresa MAGADÁN }
$$

Iconographic Rendering of the Notion of Purification in Two Elements Included in the Vignettes of Chapters 17 and 125 of the Book of the Dead Lucía DÍAZ-IGLESIAS LLANOS

Taboo - bwt?

Paul John FRANDSEN

Flat-Section Hairpins during the Egyptian Predynastic Period? A Proposal of Identification and Typology

Candelaria MARTÍN DEL RÍO ÁLVAREZ

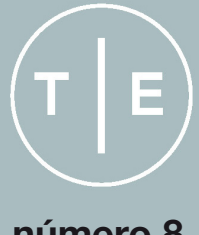

número 8 
Trabajos de Egiptología

Director

Papers on Ancient Egypt

Miguel Ángel Molinero Polo

Universidad de La Laguna, Tenerife, Islas Canarias

Secretaría de edición

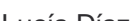

Centro Superior de Investigaciones Cientificas, Madrid

Alba María Villar Gómez

Colaborador de edición I English editorial assistant

Kenneth Griffin

Kenterity Gaino Unido

\section{Consejo de redacción}

Antonio Pérez Largacha

José Ramón Pérez-Accino

Universidad Internacional de la Rioja (UNIR)

José Ramón Perez-Accino
Universidad Complutense de Madrid

\section{Dando agua a los pájaros} Homenaje a Covadonga Sevilla Cueva

Giving water to the birds

An homage to Covadonga Sevilla Cueva

\section{Comité científico}

Marcelo Campagno
CONICET I Universidad de Buenos Aires

Josep Cervelló Autuori

Universitat Autònoma de Barcelona

María José López-Grande

Josep Padró i Parceris

Universitat de Barcelona

M. ${ }^{a}$ Carmen Pérez Die

Museo Arqueológico Nacional, Madrid

José Miguel Serrano Delgado

Evaluadores externos de este número

José Ramón Aja Sánchez | Luis Manuel de Araujo | Juan Antonio Belmonte Avilés | Elena de Gregorio |

Stan Hendryckx | Cristo Manuel Hernández Gómez | Miguel Jaramago Canora | Bill Manley | Alfonso

Martín Flores | Daniel Miguel Méndez Rodriguez | M. ${ }^{2}$ Violeta Pereyra | R. Gareth Roberts | Amelia del

Carmen Rodriguez Rodriguez | José das Candeias Sales | Inmaculada Vivas Sáinz

\section{Fundadores de la Revista}

Miguel Ángel Molinero Pol

José Ramón Pérez-Accino
Covadonga Sevilla Cueva

Editado por I Edited by

Miguel Ángel Molinero Polo | Antonio Pérez Largacha | José Ramón Pérez-Accino

Número 8

2017 


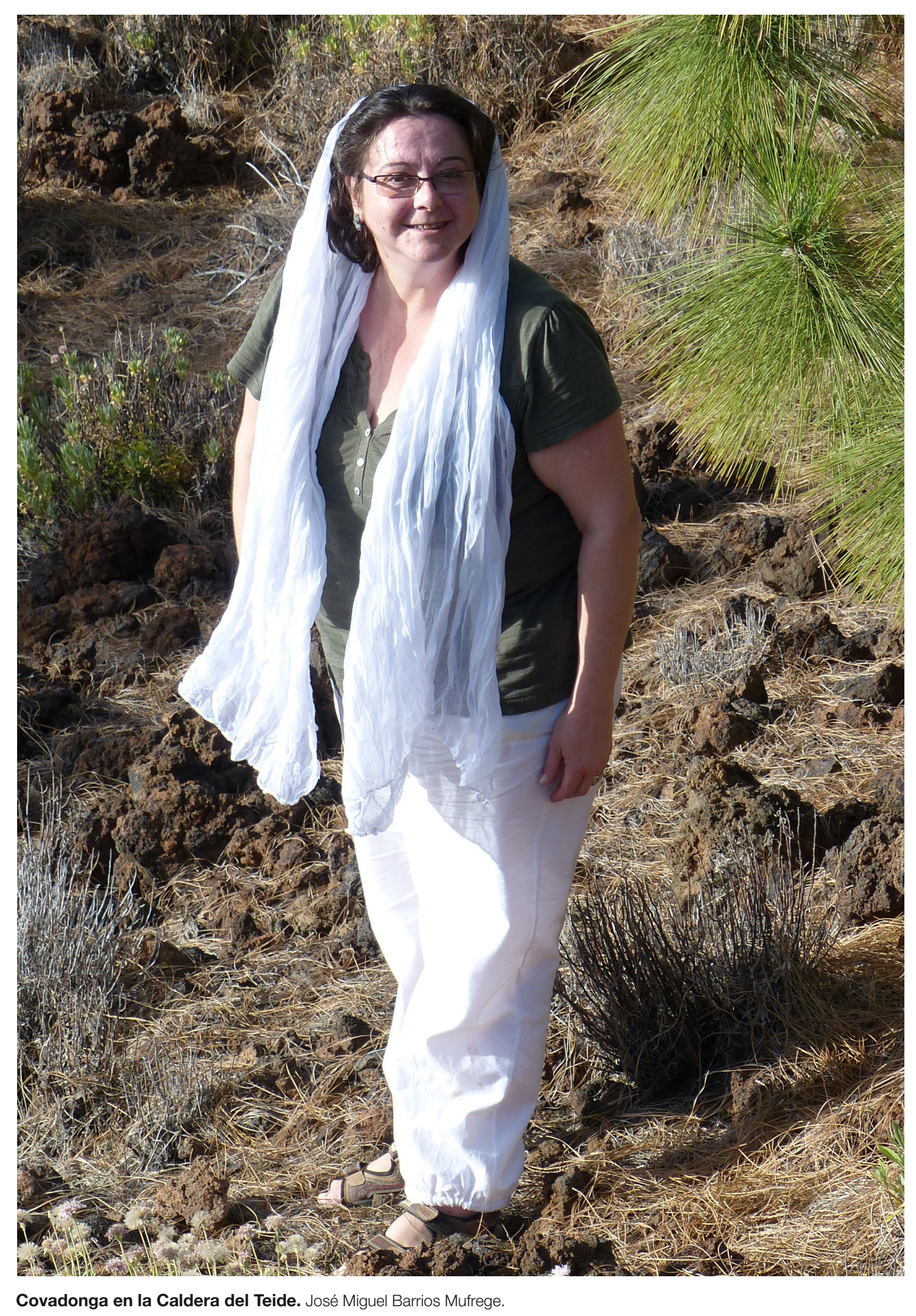




\title{
Algunas reflexiones sobre Uluburun y el intercambio comercial y cultural en el Mediterráneo oriental
}

\author{
Antonio PÉREZ LARGACHA
}

El pecio de Ulluburun ofrece la posibilidad de comprender dinámicas del comercio en el Mediterráneo en el Bronce Reciente. En la primera parte del articulo se analizan los planteamientos que sobre el destino, función y origen de la embarcación se han propuesto. Despues se examinan los pequeños objetos que transportaba y que suelen ser "olvidados" pero cuyo estudio puede en un Mediterráneo Oriental que puede ser considerado en algunos aspectos como una koiné cultural a finales del Bronce Reciente.

Some Reflections on the Uluburun and the Commercial and Cultural Exchange in the Eastern Mediterranean

The wreck of the Uluburun offers the possibility of understanding the dynamics of trade in the Mediterranean during the Late Bronze Age. In the first part of the paper, the approaches that have been proposed about the destination, function and origin of
the vessel are analysed study of these objects can provide answers to dynamics and commercial interests or policies, the exchange of ideas and share manifestations of devour
end of the Bronze Ag.

Palabras clave: Uluburun, Mediterraneo Oriental, Bronce Reciente, internacionalizacion, contactos comerciales.

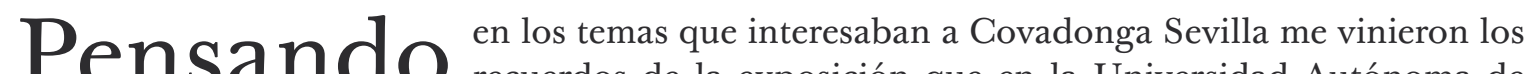
AO recuerdos de la exposición que en la Universidad Autónoma de Madrid coordinamos en torno a el-Amarna en 1991. Durante la preparación de la misma, el Prof. Luis García Iglesias conversó con nosotros sobre la importancia que podría tener el escarabeo que, con el nombre de Nefertiti, se había descubierto en el pecio de Uluburun, y sobre cómo debieron ser las dinámicas comerciales en el Mediterráneo oriental durante el Bronce Reciente. $\mathrm{Al}$ ciclo de conferencias que se organizó invitamos al Dr. Jaromir Malek y a Covadonga le sorprendió su cordialidad, naturalidad y deseos de ayudar. Estas dos personas encarnaban muchas de las facetas que Covadonga ansiaba y transmitía; conocimiento, colaboración y humanidad. Trabajando en la preparación de la exposición Covadonga disfrutó viendo cómo los alumnos de la licenciatura de Historia, o los que ya se estaban iniciando en la realización de sus tesinas, participaban con alegría, la que ella transmitía, sin egos ni aspiraciones personales. Es por ello que decidí dedicar estas páginas a reflexionar sobre algunos aspectos inherentes a la documentación hallada en el pecio de Uluburun. Este ofrece la oportunidad de reflexionar sobre las presuntas e interpretaciones que los historiadores han venido dando a unos objetos $y$ materias primas que han sido valorados por su cantidad y belleza, aś como a un la por la excecionalide estos últimos, al igual que los pequeños gestos que transmitía Covadonga, pueden comunicar más de lo que a primera vista parece.

TdE 8 (2017)- Paginas: 273 - 287

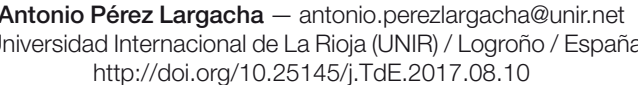




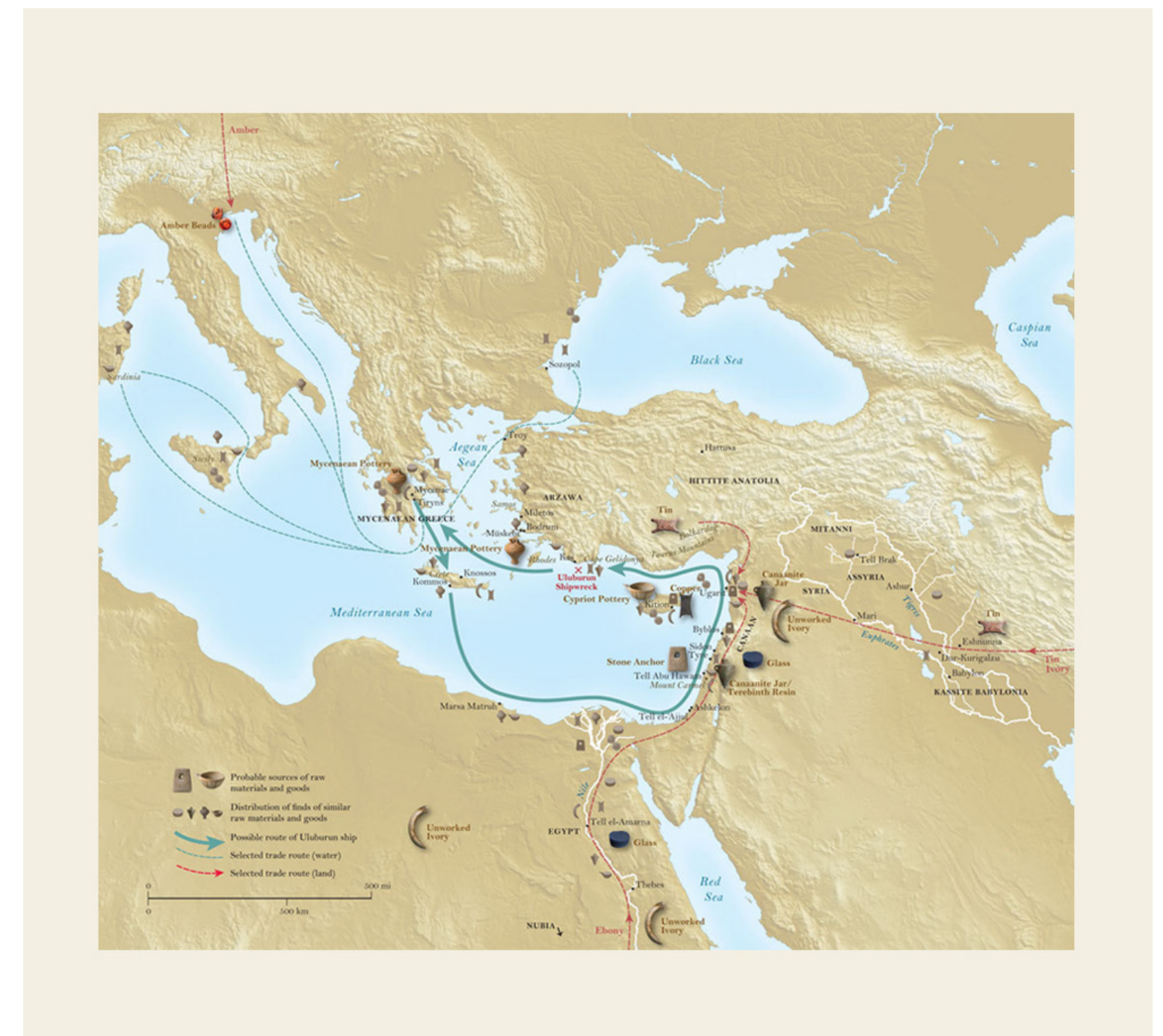

Figura 1. Posible ruta de la embarcación hundida en Ulubrun (Aruz, Benzel y Evans 2008: 298, fig. 97).

El pecio de Uluburun, excavado entre investigación la oportunidad de conocer muchos de los objetos y materias primas que una embarcación podía transportar durante el Bronce Reciente. Desde su hallazgo y los que los objetos procedieran de regiones, reinos, ciudades y culturas diferentes de todo el Mediterráneo oriental, lo que revelaba la intensidad, amplitud y organización que tuvieron las relaciones comerciales ${ }^{1}$ (fig. 1).
Hasta su descubrimiento, la documentación diplomática, las cartas que fueron intercambiadas entre las cortes palaciales del Bronce Reciente, que en muchos aspectos repetían formulas y dinámicas presentes ya en la primera edad diplomática del Bronce Medio, constituían la principal y prácticamente única fuente de información para interpretar el intercambio de bienes y productos entre los reinos de ese periodo. En ellas se menciona el papel que los embajadores y los emisarios reales tuvieron en los procesos de negociación, al tiempo que transmitían que toda la actividad comercial giraba en torno a las necesidades y demandas de los palacios y reyes, estando el comercio controlado por el Estado y centrado en torno a las deseos, requitos y demandus de los gandes deseos, requisitos y demandas de los grandes

gualmente, se destacaba de la documentación cómo los propios reyes pedían a sus homólogos que protegieran los caminos o fueran indemnizados por las pérdidas que habían sufrido las caravanas por un robo. También algunos de ellos procedían a enviar a otros reinos estatuas de dioses para proteger y ayudar en situaciones concretas, pero también artesanos, médicos o especialistas ${ }^{2}$, revelando así que todo giraba en torno a los deseos, necesidades y alianzas de los grandes reyes.

Otro elemento que aparecía en torno a los intercambios eran los llamados matrimonios diplomáticos, cuya celebacion iba unida a un intercambio zos que rinoscono el egipcio relizaban para obtener bienes exóticos de region Nubia en expect Nubia -en especial oro y marfil-, para satisfacer las demandas internas -para los templos, el

ajuar funerario y todo lo que se utilizaría en el interior de unos palacios desaparecidos- y externas. Es decir, como hemos señalado, todo apuntaba a que el comercio internacional una actividad estatal, organizada deste la a ministracion eada rinoy que no habra participión priva de la de los puncla sustantivista de Polanyi , uno de los puntos de partida para el estudio de la economía y comercio en el Próximo Oriente ${ }^{4}$ La excepción parecía estar en Ugarit, donde los textos desvelan que existían unos ricos comerciantes que disfrutaban de ventajas y privilegios para desarrollar sus empresas comerciales, no estando su actividad supervisada por la corte, aunque si seguramente apoyada para beneficio del propio reino.

Por todo ello, una de las preguntas que giró desde el primer momento en torno al pecio de Uluburun era qué entidad política podía estar detrás de su financiación y a qué corte o palacio iban dirigidos los productos que transportaba. El hallazgo de los conocidos lingotes de cobre característicos de Alashiya, así como de marfil, abundante cerámica cananea en cuyo interior se podian transportar productos variados, desde vino y aceite a objetos, colgantes, etc., todo parecía reflejar una similitud con los productos que se mencionaban en la correspondencia diplomática, por lo que se asumió que el pecio de Uluburun respondía a las dinámicas comion tes dominantes en el Bronce Reciente.

En relación con la datación del pecio, desde su hallazgo se aceptó que ocurrió en torno al 1300 a.C., existiendo pocas variaciones en la actualidad, como la fecha del 1320 a.C. propuesta por Manning et alii ${ }^{5}$. Una época en la

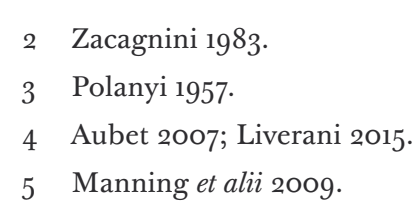


que es importante tener en consideración el contexto histórico, debido a la cantidad de actores que, de una fo llegaron a intervenir ecimiento de la embarcación, los productos que translaba y su posible destino.

Igualmente, tampoco puede olvidarse que el comienzo del siglo XIII implicó en el Mediterráneo oriental el diseño de un nuevo mapa internacional, debido a los cambios que había originado la irrupción del reino hitita en Siria y la desaparición del reino de Mitanni. La importancia de tener en cuenta el contexto histórico global a la hora de analizar un reinado, un hecho histórico o un acontecimiento está perfectamente reflejada en la obra de van de Mieroop ${ }^{6}$ quién defiende la necesidad de investigar, interpretary valorar no solo de láptica de la egiptolos cluso, desde la optica de reinos más pequen cluso, desde la optica de reinos más pequeños como el de Ugarit, es decir, eliminar barreras académicas y cientificas que la historiografía ha establecido en torno a culturas y regiones cuyos intereses, comerciales y políticos, estaban interrelacionados

En Egipto estaba teniendo lugar el tránsito de la XVIII a la XIX dinastía, después de un período amarniense que, además de sus connotaciones religiosas, había vivido cambios sustanciales en la influencia y control que el mundo faraónico ejercía sobre el Levante mediterráneo debido al expansionismo de Suppiluliuma I. Unos a nos en los que el teino Suppiluliuma I. Unos años en los que el reino

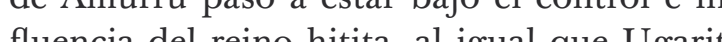
fluencia del reino hitia, al igul que Ugart, uno de los principales puertos comerciales de

Mediterráneo y donde la embarcación hundida en Uluburun pudo obtener parte de su caramento. Tampoco debe olvidarse que Ugarit se convirtí en uno de los aliados más feles del rino hirita, lo que en teora pudo teles

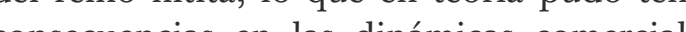
consecuencias en las dinámicas comerciales que el mundo egipcio quería mantener con Levante. Igualmente, Asiria intentaba recuperar territorios que anteriormente habian estado bajo su influencia aprovechando la desaparición del reino de Mitanni, lo que iba originar una atmosfera de conflicto y recelo constante con Hatti. Por su parte, la Babilonia casita recuperaba lentamente un esplendor pasado al tiempo que debía prestar una atención cada vez mayor a Irán.

En el ámbito del Egeo y el Mediterráneo oriental Chipre continuaba siendo un centro distribuidor de cobre y se hallaba en medio de Pero el cambio ús importante fue el auge de

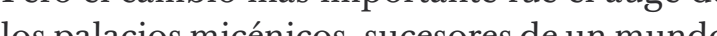
minoico alificanicos, sucesores de un mundo na "to cacio". Al mismo

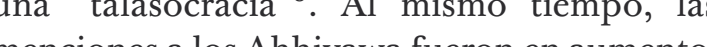
menciones a los Ahhiyawa fueron en aumento, debido a sus conflictos con el mundo hitita precisamente en unas regiones que, en principio, afectaban a las vías de comunicación marítimas de Asia Menor, la ruta de cabotaje que parecía seguir la embarcación hundida en Uluburun.

Una embarcación que tenía 15 metros de arcoyuna En opinión de Gestoso Singer ${ }^{10}$ debí ser una encón dose en la creencia de Sherratt y Sherratt ${ }^{11} \mathrm{de}$ que las embarcaciones mercantes tenían una capacidad de unas 225 toneladas.

En cualquier caso, su capacidad de carga imPlica un gran esfuerzo económico y logítico. Al respecto, $\mathrm{Monroe}^{12}$ ha analizado las perdidas corción y de los productos que tranpora Aun siendo difici poder cuanticar el valor de Aun siendo dificil poder cuantificar el valor de a productos -además de todos aquellos que se han perdido arqueológicamentela estimacion de unos 12.000 shekels proporciona una imagen de una gran pérdida económica con relación a todo lo que podría obtenerse con la venta y posterior elaboración de las materias primas que transportaba en los talleres artesanales a los que iban dirigidas dichas materia primas. También deben considerarse todas las pérdidas ocasionaden en la pas peción, búsqueda y obtención de los objeso-

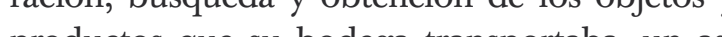
productos que su bodega transportaba, un

pecto que en pocas ocasiones se contempla.

En relacion con su destino, parece que er algún puerto del Egeo. La mayor parte de la cerámica hallada es del Levante, siendo la de Egeo escasa, al tiempo que la misma presenta signos de haber sido muy utilizada, lo que puede reflejar que era la utilizada por los miembros de la tripulación, no estando destinada al comercio o intercambio ${ }^{13}$

Respecto al puerto de origen pudo estar en el Levante quizás en Ugarit, pero lo que sorprende es la gran cantidad de lingotes de cobre de Chipre, lo que hace pensar que antes de gar al Levante la embarcación realizaría una esala en Chipre.

En líneas generales, se acepta la idea de que la embarcación iría recogiendo en su trayecto

2 Monroe 2010

3 Bachhuber 2006: 34

14 Kilian 1993

15 Cline y Yasur-Landau 2007: 128.

6 Pulak 200 objetos y productos que después serían intercameran cim implica un conoy los productos que podía existía en cada luga los productos que podran obtene, asi como ye revizar In se realizarian intercambios que, como veremos, pudieron ser de un carácter oficial e instituciona pero también privado. Una de las preguntas que es difícil poder contestar es qué tipo de productos transportaría en un principio la embarcación antes de llegar a Chipre o si, por el contrario, los productos de Chipre serian comprados, lo que implicaría que detrás de la expedición estarí una entidad política que sufragaba los gastos.

Éste es precisamente uno de los debates existentes en torno a la embarcación. ise trataba de una misión diplomática de una expedición organizada por una institución (palacio, reino) o, por contrario, puede esta embarcán estar refejando una actividad privada, aunque fuese con apoyo institucional?

$\mathrm{Al}$ respecto, Kilian ${ }^{14}$ apuntó la posibilidad de que se tratara de la embarcación de un rico mercader basándose en la importancia, rique za y autonomía que los mismos tenían en especial en el reino de Ugarit. Sin embargo, en opinión de Cline y Yasur- Landau ${ }^{15}$, la expedición ue seguramente palacial debido a los costes, precio y los peligros a los que debió enfrentarse. Así, obtener once toneladas de metal -diez de cobre y una de estaño-, supondría un esfuerzo que solo puce interpretarse si hab́r un zo que solo puede in la expedicion. Por su parte, Pulak ${ }^{16}$ defiende que formando parte de paripular habra, al menos, dos micen que podrían haber actuado de la misma form 
que posteriormente lo haría el sacerdote egipcio Wenamun, comprando y negociando en los puertos en nombre y representación de una entidad política, que en opinión de Cline y Yasur-Landau podrían ser Kommos, uno de los puertos más importantes de Creta, o bien el palacio micénico de Tirinto.

En opinión de Bachhuber ${ }^{17}$, estaríamos ante una manifestación de un intercambio entre elites, pero no necesariamente se trataría de regalos reales al poder estar destinados algunos productos a consumidores no palaciales. Si bien esto último puede ser cierto, también lo es que los palacios actuaban como centros receptores que posteriormente procedían a trabajar y a repartir los productos para su elaboración, consumo o disfrute, por lo que palacio estaŕa de alguna forma implica que

El origen diverso del cargama is or diferentes puetos también explica la pre por diferentes puertos también explica la presencia de tres balanzas y 149 pesas, siendo significativo que ninguna pueda adscribirse al sistema de pesos y medidas micénico, un argumento más para defender que el destino final de la embarcación estaría en el Egeo.

Como ya hemos apuntado, la variedad geográfica de los objetos y productos siempre se ha destacado, desde el ámbar del Báltico al marfil africano, pero uno de los aspectos que sorprende, a primera vista, es la escasez de objetos egipcios. Fllo lleva a plantearse la posibilidad de que los mismos podín no in posio que Egipto se encontaba desplaza do los - qua Egipto se encontraba desplazado de los circuitos come habín acontecido en el Levare polticos que hab́an acontecido en el Levante con la expansión de Hatri y que obligaron a los faraones de la XIX dinastía a emprender

una política más militarista para recuperar su influencia en la región y poder continuar participando en los intercambios comerciales del Mediterráneo oriental. Una tercera posibilidad es que los mismos hubieran sido cober lán purto del Levante. En relación co en ausen teó la de Ugarosis de que la ruta del barco era de Ugarit a Egipto pero que vio su trayecto desviado por los fuertes vientos, lo que parece poco probable.

Sin embargo, todo parece indicar que la embarcación si realizó una escala en Egipto. No por el ya mencionado escarabeo de Nefertiti, que nos sirve para comprobar como en muchas ocasiones un hallazoo se interpreta en función de lo que resulta más "llamativo" y que, como veremos, pudo llegar a la emon barcación por or arte del carganento se hallaron lingotes de vidrio, siendo los primeros que se conocen y que, a juzgar por los análisis realizados al menos en tres de ellos, son de procedencia egipcia ${ }^{19}$

Esta procedencia egipcia es muy reveladora, ya que la industria del vidrio fue introducida en Egipto desde el mundo mesopotámico posiblemente durante el Segundo Período Intermedio, conociéndose diferentes centros productores en el Reino Nuevo en Amarna o Qantir ${ }^{20}$. Su hallazgo también revela que toda la materia prima transportada estaba destinada a ser trabajada en los talleres, y por arteanos del reino de desino, posible artede el dios de Walton e ali:21 dios de Walton eluine en el sentido de que a procedencia del vidrio hallado en algunos

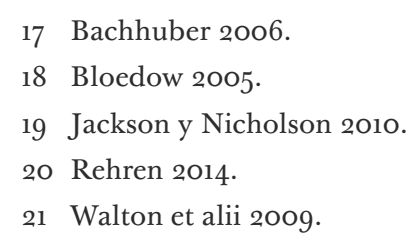

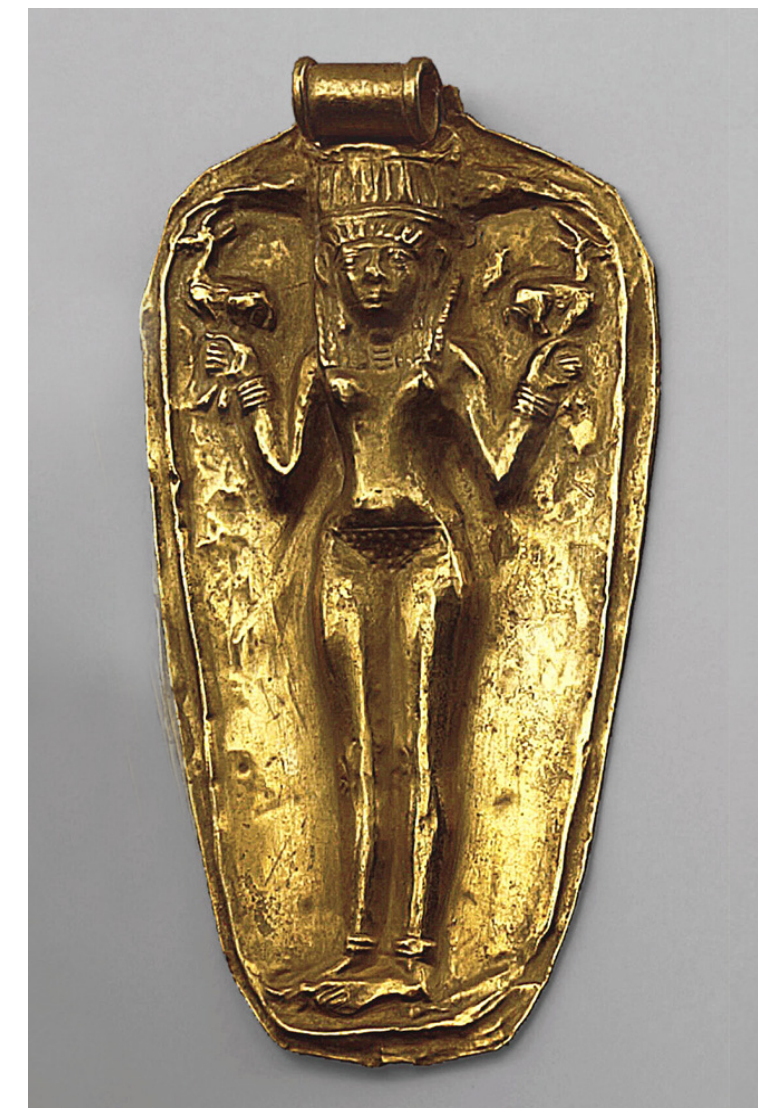

bilidad en el que se irían anotando los intercambios que se realizarían durante la travesía, así como los objetos embarcados para su destino final. Ésta sigue siendo la interpretación dominate, pero rion señado que en realida podra señalado que en realitad podria tratarse de un pasaporte, o incluso de un menaje para que fuera leído en el lugar de destino, no un libro de cuentas.

Otra de las consecuencias del hallazgo de este pecio es que sirvió de base para que se comenzara a hablar de un "estilo internacional", término acuñado por Stevenson Smith ${ }^{23}$ para referirse a unos objetos cuya iconografía y función no podía adscribirse a un reino o estado concreto. Los mismos nos transmiten la existencia de unos gustos que eran similares entre las clases dirigentes, de una koiné cultural que reconocía apreciaba y utilizaba símbolos, actitudes y temáticas similares, po simdo las bases de lo que despés seŕa un po do las bases de lo que despues sería una de caracteristicas del mundo fenicio y la difusión de objetos, escenas y objetos con temáticas orientales por todo el Mediterráneo. Unos objetos que transmitían unas ideas, sentimientos y valores que eran conocidos en todo el Mediterráneo Oriental ${ }^{24}$.

También con relación al contexto del pecio palacios micénicos era egipcia. Igualmente, si estos lingotes de vidrio fueron embarcados en Egipto nos está revelando una intencionalidad, el buscar un producto que se demandaba, como sucedía con los lingotes de cobre de Chipre, al tiempo que abre nuevas interpretaciones sobre las industrias y tecnologís desaciones sobre las industrias y tecnologias desaUladas en Egipto.

Uno de los objetos más sorprendentes y llamativos hallados en Uluburun es el que se consideró desde el inicio como un libro de conta-

de Uluburun hay que tomar en consideración los argumentos esgrimidos en las últimas décadas en el sentido de que a lo largo del siglo XIII a. existió una actividad com siglo que no grandes reinos, un comercio no estatal.

Sin poder entrar a fondo en este nuevo marco y debate, debemos mencionar al menos os planteamientos de $\mathrm{Artzi}^{25}$ sobre la existencia de unos comerciantes que se dedicaban al 
reciclaje de metales que después comerciaban y cuyas actividades pueden ponerse en relación con los cambios estructurales del s. XIII a.C. y los posteriores Publos del Mar. Xambién los de Sherrate ${ }^{26}$ Pueblos del Mar. También los de sherte sobre la existencia de una actividad comercial fuera de los ámbitos palaciales que seria, posteriormente, una de las causas de las crisis del 1200, atribuida historiográficamente a los Pueblos del Mar pero cuyos movimientos, así como la crisis de las estructuras palaciales, podían ponerse en relación con un cambio en las dinámicas económicas. En esta línea, Singer ${ }^{27}$ sugirió que Uluburun puede interpretarse como una consecuencia de la apertura de rutas, mercados e intercambios de las grandes potencias con anterioridad al 1300 a.C, siendo reflejo de un comercio más dinámico.

En definitiva, si se confirman estas dinámicas implicaría que la embacacion de Uluburun y el comercio del Mediterráneo oriental desarrollarían sus actividades en un mundo en cambio y direcciones opuestas.

\section{Reflexiones en torno a los objetos}

\section{pequeños}

Cuando se habla de comercio siempre suele hacerse en términos de los objetos y productos que son intercambiados, pero todo objeto tiene una historia, una biografía cultural ${ }^{28}$ que permite profundizar en su significado y realiza funcion determing un función deterninada y ser hallado en un con-

Otro aspecto que explorar es que detrás de todo comercio e intercambio de objetos también está el tránsito de ideas y personas. cos, con su celebración se trasladaba un sćq to que iba a vivir en otro reino, en su séquito que iba a vivir en otro reino, en su corte o , pera que tendríaría eque sequito asimilaría aspectos culturales del reino al que se trasladaba. Unos sirvientes, traductores y artesanos, personas que viajarían con sus amuletos y adornos personales, buscando la protección de sus divinidades personales o las que encarnaban su memoria cultural y que, de ese modo, junto a los comerciantes, mercenarios, artesanos itinerantes..., transmitían unas concepciones nuevas que, en algunos casos, podían llegar a ser adoptadas tanto en el ámbito oficial de la corte como fuera de ella. Esta es la importancia de los objetos pequenos, de las personas que viajan y se trasta dan. Con ellos vinjan ideas, creencine genes que es difíl encontrar en ámbito ó cial o diplomático y que, cuando aparecen cial o diplomático y que, cuando aparecen en de una es posiblemente como consecuencia de una aceptación de las mismas debido a su expansión en determinados círculos relacionados con la corte.

Por otra parte, los marineros, como los comerciantes, podrían reunir, comprar, adquirir objetos en los diferentes puertos o ciudades por los que transitaban, bien por un interés personal o incluso para comerciar con ellos en sus ciudades y reinos de origen. Esta puede ser la explicacion, por ejemplo, del escarabeo de Nefertiti.

Un único escarabeo, o cualquier otro objeto con una inscripción puede interpretarse, utilizando parámetros tradicionales, como una forma de datación para fijar el contexto del conjunto. Sin embargo, en el caso del escarabeo de Nefertiti pudo ser adquirido por algún miembro de la tripulación como un recuerdo o con la idea de comciar despues con él en su propia ciudady reino. Lógicamente mismo tendría un valory significido en tiempos de Amarna pero con posterioriad desapareció más allú de los ele postrionidad desapareció más allá de los elementos egipcios que hay en el mismo, al igual que sucede en otros muchos ejemplos, por lo que su presencia en Uluburun puede no responder al ámbito oficial. Otra pregunta o posibilidad es que ese escarabeo pudo no ser adquirido en Egipto, sino en cualquiera de los puertos del Levante ya que estos objetos son fácilmente transportables y además fueron utilizados como medios de difusion fuera de la corte y del mundo dios de difusion fuera de la corte y del mundo

Pequeños objetos, como sellos o amuletos, sobre los que pueden aducirse dos posibilidades;

A) Eran pequeñas posesiones que pertenecían a los miembros de la embarcación, que buscaban en ellos una protección personal ante los peligros que implicaría desplazarse, mantener una vinculación con sus divinidades personales. En estos casos, los mismos podrían en tenderse en opinion de $\mathrm{Pulk}^{29}$, codó de la identide de de la identidad de sus propictarios, siendo as que al menos dos miembros de la tripulación eran micénicos que pudieron estar incluso relacionados con la administración palacial para garantizar y obtener los productos que estaban destinados al palacio.

B) Pueden estos objetos estar reflejando también la transmisión de ideas, creencias y gustos entre poblaciones fuera del ámbito diplomático, un pequeño comercio que realizaban tripulantes aprovechando la coyuntura.
En relación con estos objetos, su manufactura no es muy elaborada, lo que implica otro aspecto; cuando se piensa en comcio e intercambio lo hacemos en términos de bellos y valiosos objetos, pero fuera de los ifcuitos oficias exín muchas for de borato de a do o a dornos que estaban destinados al menos a un porcentaje de la población. Unas manife taciones artísticas calificadas de "artes menores", relegándolas en muchas ocasiones al olvido o, en el mejor de los casos, a ser consideradas curiosas, exóticas ${ }^{30}$

En época orecorromana las monedas transensajes e ideas, así como otros pequeños objetos adscritos a comercintes, solda o artesanos. Lo mismo pudo suceder en un Bronce Reciente interrelacionado, complejo en las relaciones y que, además, es importante recordarlo, tenía un trasfondo que ya se había desarrollado en el Bronce Medio. Al respecto las reflexiones de $\mathrm{Aruz}^{31}$ sobre las dinámicas de difusión y transmisión de los cilindros sellos y sus motivos son muy reveladoras.

Unos objetos fácilmente transportables, que transmitían símbolos e imágenes de mundos $y$ culturas diferentes, que llamaran tención cuando eran contemplados por la población local. Lo mismo sucedería con sus vestidos, de los que, desgraciadamente, apenas conocemos restos o representaciones, pero cuyos colores, dibujos... realzarían los peinados característicos de cada región y cultura, los adornos personales, pendientes, brazaletes, amuletos o broches. Todo ello iría generando, como se ha mencionado desde el Bronce Medio sino antes, que los símbolos fueran perdiendo su iconografía original, que 
algunas divinidades se representaran de una forma que fueran reconocibles para todos, tanto en tanto en sus actitudes,

El problema es querer identificar todo objeto, o su decoración y motivos, con etnicidad, entrando en muchas ocasiones en debates estériles que poco aportan. Desde el Bronce Medio hay un flujo constante de temáticas, imágenes, expresiones y objetos que hace difícil poder determinar el origen del objeto $o$, incluso, del motivo, que va aglutinando detalles y características de mundos diferentes.

Es en este contexto en el que se pueden incluir también las cuentas, objetos que desdo Neolítico acompañn a los muertos y que nos revelan la existencia de un inters por obtener materales como el lapićati por obeque tranias cono el lapisázuli o conchas que transmitfan unas creencias, pero también un comercio, un intercambio que podía ser realizado por poblaciones nómadas en contacto con las sedentarias. Así, en el caso de Uluburun resulta muy reveladora la presencia de 75.000 cuentas de fayenza y 9.500 cuentas de vidrio en el interior de cerámicas cananeas que, según su capacidad (27 litros), podín llegar a contener hasta 26 . podin que, segin su copacidad (27 cada una lo qu mportancia.

Otros dos ejemplos de restos hallados en Uluburun acercan a un mundo diferente a de las cartas diplomáticas. El primero de ellos es un molar de un ratón, cuyo análisis ha permitido ponerlo en relación con especies do- cumentadas en Minat el Beida ${ }^{33}$. El segundo ejemplo son las significativas cantidades de resina halladas ${ }^{34}$ un producto que es dif́cil de de constar arqueologicamente. Ello indica que, además de todos los productos que son mencionados en los textos en el Mediterráneo oriental, se comerciaba con muchos otros que también tenían un valor y consideración ${ }^{35}$, lo que hace años se denominaron "exportaciones invisibles".

Objetos pequeños que ofrecen otra visión del comercio ajena al ámbito diplomático de las cartas. Igualmente, los mismos indican cómo ideas y sentimientos comunes en regiones muy diferentes pueden manifestarse en una repesente caso con a to de un pendiente de do en Uluburun (fig. 2)

Bass $e t a^{3 i i^{36}}$ lo asociaron al grupo de representaciones que se conocían de la diosa del había realizado $\mathrm{Negbi}^{37}$. Se trata de un pendiente de forma piriforme que representa a una mujer desnuda con sus pies orientados hacia la derecha -lo que es inusual-, sujetando con sus manos unas inclo to que solo colo ecosedentes de Ugarit. Esta actitud se ha relacionado con el motivo de la Señora de los Animales, llevando una corona cilíndrica pero no el caser el que llevan este tipo de figuras ${ }^{38}$. Unos pendientes que, por la representación de una Levante Qudsu, basándose en la tipología que racterístico peinado hathórico, que suele

32 Ingram 2014.

33 Cucchi 2008.

34 Pulak 2008.

35 Papadopoulos 2012

36 Bass et alii 1989: 2, fig. 3

37 Negbi 1976.

$3^{8}$ Pulak 2008: 347 . mujer desnuda relacionada con diversos símbolos de poder y divinos, se han relacionado con diosas vinculadas a la fertilidad $y$, en últimos años, también con el erotismo.

La representación de figuras femeninas desnudas se remonta al IV milenio. Incluso con anterioridad, toda figura femenina en que los rasgos sexuales están remarcados siempre se han vinculado con la fertilidad. Es decir, la imagen y finalidad de estas imágenes no cambia con el paso del tiempo, pero sí lo hacen los atributos y formas de ser representadas.

Debido a nuestras concepciones y deseos, en la mayoría de las ocasiones queremos identificar lo que se representa, ponerle un nombre y, de ese modo, explicar su aparición, función, adscribirla a una cultura y región o determinar ana posible influencia difusión. Esta actitur una posible influd de categorizaion que, en mulas ocasio de categonzación que, en michas ocasiones, domina tanto la interpretación arqueológica historica y que ocasiona que nos olvidemos de realizar otro tipo de preguntas al objeto para poder llegar a conocer realmente su función aparición, difusión y significado.

En el Bronce Reciente son muy frecuentes estas figuras femeninas y la actitud de las mismas, estén desnudas o no, teniendo en general los brazos en V, en muchas ocasiones asentadas sobre un león y sosteniendo en sus manos animales -como en el caso del pendiente de Uluburuno bien otro tipo de objetos, desde armas a seo bien on tho de objetos, desde armas a sc

En Mesopotamia la diosa Ishar desde En Mesopesde tiempos del reino acadio se representa con alas, como los genios, pudiendo transmitir las mismas un mensaje de protección y relación con el mundo de los dioses. Una diosa que en el ámbito sirio-palestino será conocida como

39 Sugimoto 201

40 Budin 2015

41 Negbi 1976: 95-7
Astarté, una divinidad con la que también se han querido identificar estas representaciones femeninas que aparecen en pendientes o terracotas. En tiempos posteriores, será Afrodita la cotas. En tiempos posteriores, será Afrod

Como en tantos otros aspectos, los orígenes de estas figuras sirio-palestinas del Bronce Reciente pueden buscarse en el Bronce Medio. Es cierto que es difícil establecer una línea de continuidad. Ello se debe, por un lado, a la compartimentación "aristotélica" que hemos realizado de la Historia y los objetos que caracterizan cada periodo y, por el otro, al hecho de que a medida que las relaciones e intercambios (comerciales, de personas e ideas) aumentan, los objetos y manifestaciones van cambiando sus características, al tiempo que van siendo adaptados a las creencias y costumbres de cada región.

Igualmente, pero no menos importante, estamos ante objetos pequeños, que aparecen en contextos arqueológicos muy diferentes y, en líneas generales, no están asociados a la corte, a las élites. Es por ello que reciben el calificativo en ocasiones de "populares" lo que, en muchas ocasiones, lleva implícito una minusvaloración de la información histórica y cultural que nos pueden proporcionar.

La figura representada en el pendiente de Ulu Burun se ha identificado con Qudsu, divinidad que también se ha identificado con Qedeset, divinidad conocida iconográficamente en Egipto a partir del reinado de Ramsés II. Esto hace pensar a Budinado de (and que de paz fircto cos aunque considera que Qudsu y Qedeset fueron dos divinidades diferentes.

La mayoría de los pendientes, 18, están realizados en oro ${ }^{41}$, por lo que, en general, se han 
adscrito a una clase social concreta, al tiempo que se han asociado a cultos licenciosos, sórdidos o a diosas como prostitutas sagradas ${ }^{42}$. Como apunta Benzel ${ }^{43}$, en muchas ocasiones los estudios se han centrado en "quién" se representa, no en el "qué", señalando que su presenta, no en el qué, señalando que su
desnudez las acerca más al culto privado, no al oficial que es más abstracto. Este hecho, unido a unas técnicas de producción no muy elaboradas, le hace pensar más en un culto privado, no oficial como piensa Pulak ${ }^{44}$

En cualquier caso, e independientemente de origen de las tradiciones, la imagen de una diosa vinculada con la fertilidad, prosperidad y protección es innegable. Pueden cambiar los peinados, los atributos o símbolos que sostienen en las manos, pero todas ellas serían recon Existe una mente un ide general sobre la que postriorḿs locies, pero todo lloconfina der qus habra pero todoelloconfirma y demuestra erráneo oriental ${ }^{45}$.

\section{Conclusiones}

Los objetos pequeños suelen ser poco valorados, en ocasiones por su tamaño, por aparecer en contextos arqueológicos poco claros, poder adscribirse a diferentes períodos en función de la iconografía, símbolos o actitudes de las figuras representadas, el material con que están realizados... Principalmente, esto se debe a que no se mencionan en las cartas diplomáticas y en los textos, al tiempo que estos mismos objetos no tienen, en la mayoría estos mismos objetos no tienen, en la mayoría
de las ocasiones, un nombre o un signo que de las ocasiones, u
pueda identificarlas.

$$
\begin{aligned}
& 42 \text { Bahrani } 1996 . \\
& 43 \text { Benzel 2013: } 258 \text {. } \\
& \text { 44 Pulak 2008: } 347 \\
& 45 \text { Cornelius 2008. }
\end{aligned}
$$

Sin embargo, serían estos objetos los que de forma mayoritaria se moverían entre las poblaciones y culturas. Junto a los lingotes de cobre podemos encontrar miles de cuen-

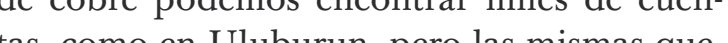
tas, cono quedan relegadas en la función e importancia que podfan tener en las relaciones en la transmisión de ideas o mensajes, la existencia de unos gustos comunes o de unos símbolos que transmitirían las mismas preocupaciones y deseos.

Uluburun, como otros pecios más pequeños tambien del Bronce Reciente, ofrecen la posibilidad de obtener una visión de conjunto, global. Aun así, es difícil que no nos centremos en los objetos y materiales a los que nos acostumbran los textos, quizás por la concepción aristotélica mencionada y también, por nuestra mentalidad museística coincidente con el redescu glos XVIII XIX. Igualmente, to dinvestiga dor quiere hallar, aportar algo que tenga "repur a las gusión", pero no debe olvidar que junto multitud de objetos, símbolos, materiales, productos... que, en conjunto, no hacen, sino que valoremos más lo que del pasado conocemos, como Covadonga lo hacía.

\section{Bibliografía}

ARTZI, M

2013 "On the Other Sea Peoples", en: A.E. Killebrew y G. Lehmann (eds.): The Phichaeology (Society of Biblical Literature 15), Atlanta: 329-344.
ARUZ, J.

2013 "Seals and the Imagery of Interaction" en: J. Aruz, S. Graff y Y. Rakic (eds.): Cultures in Contact. From Mesopotamia to the Mediterranean in the Second Milennium BC, New York: 216-225.

ArUZ, J.; Benzel, K.; Evans, J. (eds.)

2008 Beyond Babylon. Art, Trade and Diplomacy i he Second Millennium B.C. New York.

Aubet, M. ${ }^{a}$ E.

2007 Comercio y colonialismo en el Próximo Oriente antiguo. Los antecedentes coloniales del II

Bass, G.; Pulak, C.; Collon, D.; Weinstein, J. 1989 "The Bronze Age Shipwreck at Ulu Burun. 1986 Campaign", AfA 93 / 1: 1-29.

BACHHUBER, C.

2006 "Aegean Interest Ulu Burun Ship", AfA 110/3: $345-363$.

BAHRANI, Z.

1996 "The Hellenization of Isthar: Nudity Fetishism and the Production of Cultura Differentiation in Ancient Art", Oxford Art fournal $19 / 2: 3-16$.

BELL, C.

2011 "The Merchants of Ugarit: Oligarchs of the Late Bronze Age Trade in Metals", en: V. Kassianidou y G. Papasavvas (eds.): Eastern Mediterranean Metallurgy and Me ford: 180-187.

Benzel, $\mathrm{K}$.

2013 "Ornaments of Interaction: Jewellery in "Y Late Bronze Age", en: J. Aruz, S. Graft Yesopotamia to the Mediterranean in the Second Millennium BC, New York: 258-266.

Bloedow, E.

2005 "Aspects of Trade in the Late Bronze Age Mediterranean: What was the Ultimate Destination of the Uluburun Ship?", $A e$ gaeum 25: $335-341$.
Budin, L.

15 "Fertility and Gender in the Ancient Near East", en: M. Masterson, S. Rabinowitz y R. James (eds.): Sex in Antiquity. Exploring Londer and $30-49$

Cline, E.; Yasur-Landau, A.

Musings from Distant Shore. Nature and go", Tel Aziv 34 / 2: 125-141.

CoRnelius, I.

2008 The Many Faces of the Goddess. The Iconography of the Syro-Palestinian God-desses Anat, Astarte, Qedeshet and Asherat c. $1500-1000$
$B C E(O B O 204)$. Fribourg, Göttingen.

Cucchi, T.

2008 "Uluburun Shipwreck Stowaway House Journey", Fournal of Archaeological Science $35: 2953-2959$.

Feldman, M.

2006 Diplomacy by Design: Luxury Arts and an International Style in the Ancient Near East $1400-$
1200 BCE. Chicago.

2007 "El barco naufragado en Ulu Burun y el intercambio de bienes en el Mediterráneo Oriental", Davar Logos 7: 19-32.

Gosden, C.; Marshall, Y. 999 "The Cultural Biography of Objects", World
Archaeology 31 / 2: 169-178.

2014 "Vitreous Beads from the Uluburun Shipwreck", Polish Archaeology in the Mediterranean 23/2: 225-245

JACKSON, C. NICHOLSON, P.

2010 "The Provenance of Some Ulu Burun Glass Ingots", Fournal of Archaeological Science 37 295-301.

KELDER, J.

Kingyawa and the World of the Great "ings. A Re-evaluation of Mycenaean Po-

016 "Een Paspoort uit de Late Bronstijd?", Tijdschrift voor Mediterrane Archeologie 28: 1-7. 
KILIAN, I.

1993 "Überlegungen zum spätbronzezeitlichen Schiffswrack von Ulu Burun (Kas)", museums 40: $333-35^{2}$

KNAPP, A.

1993 "Thalassocracies in Bronze Age Eastern Mediterranean Trade: Making and Breakorld Archaeology 24 / 3: 332-

LIVERANI, M.

2015 "Exchange Models in Historical Perspective", en: B. Eder y R. Pruzsinsky (eds.) Political Systems and Modes of Interaction in the Aegean and the Near East in the 2nd Millennium BCE, Viena: 19-31.

Manning, S.W.; PUlak, W.; Kromer, C.;

Talamo, B.; Bronk, S.; DeE, M.

2009 "Absolute Age of the Uluburun Shipwreck: a Key Late Bronze Age Time-Capsule for the East Mediterranean", en: S.W. Kinning y M.J. Bruce (eds.): Tree-Rings, ronment Papers Presented in Honour of Peter Ian Kuniholm, Oxford: 163-187.

Monroe, C.

2010 "Sunk Costs at Late Bronze Age Uluburum", BASOR 357: 19-33.

\section{NegBi, O.}

1976 Canaanite Gods in Metal. An Archaeological Study of Ancient Syro-Palestinian Figurines.
Tel Aviv.

Papadopoulos, A.

2012 "A Matter of Quantity? Some Notes on Late Bronze Exchanges Modes in the

Pérez Largacha, A.

1998 "Egipto y el Próximo Oriente. Perspectivas de trabajo y colaboración", ISIMU 1: $247-254$

PolanYi, K.

1957 Trade and Markets in the Early Empires. New York.
PULAK, C.
1988 "The Bronze Age Shipwreck at Ulu Bu"Tun, Turkey. 1985 Campaign", AfA 92: 1-37.

1998 "The Uluburn Shipwreck: An Overview", International Journal of Nautical Archaeol-

ogy 27/3: 188-224.
2001 "The Cargo of the Uluburun Ship and Evidence for Trade with the Aegean and Beyond,, en: L. Bonfante y V. Karageorghis
(eds.): Italy and Cyprus in Antiquity $1500-$ (150 B.C. Nicosia: 13-60- in Anitying $1500-$

450 B.C., Nicosia: 13-60.
2005 "Who Were the Mycenaeans Aboard the Greco (eds.): Emporia tral and Eastern Mediterranean: 295-312.

2008 "Pendant with Nude Female", en J. Aruz, K. Benzel y J. Evans (eds.): Beyond Baby-
lon. Art, trade and Diplomacy in the Second Millennium B.C., New York: 347-348. ReHRen, T.

2014 "Glass Production and Consumption between Egypt, Mesopotamia and the Aenicka, S. Lange y T. Kaster (ed.): Contextualising Grave Inventories in the Ancient Near East (Qatna Studien Supple-menta 3), Wiesbaden: 217-223.

Sherratt, $\mathrm{S}$

2003 "The Mediterranean Economy: Globalization at the End of the Second Millennium BCE", en: G. Dever y S. Gitin (eds.): Symbiosis, Symbolism and the Power of the Past, Indiana: 37-62.

Sherratt, S.; Sherratt, E.

1991 "From Luxuries to Commodities: the Nature of Mediterranean Bronze Age TradTrade in the Mediterranean, Jonsered: 35186.

Stevenson Smith, W.

1965 Interconnections in the Ancient Near East. Yale.

Sugimoto, D. (ED.)

2014 Transformation of a Goddess. Isthar-AstarteAphrodile ( $O B O$ 263). Freiburg, Gottenburg.
THOMAson, A.

2014 "The Impact of the Portable: Integrating "Minor Arts" into the Ancient Near Eastern Canon", en: M. Feldman y B.
Broen (eds.): Critical Approaches to Ancient Near Eastern Art, Berlin: 133-157.

VAn de Mieroop, M.

2007 The Eastern Mediterranean in the Age of Ramesses II. Oxford.

Walton, M.; Shortland, A.; Kirk, S. DEGRYSE, $\mathrm{P}$

2009 "Evidence for Trade of Mesopotamian and Egyptian Glass to Mycenaean 36: 1496-1503.

ZACCAGNINI, C.

1983 "Patterns of Mobility among Ancient Near Eastern Craftsmen", JNES 42: 245- 


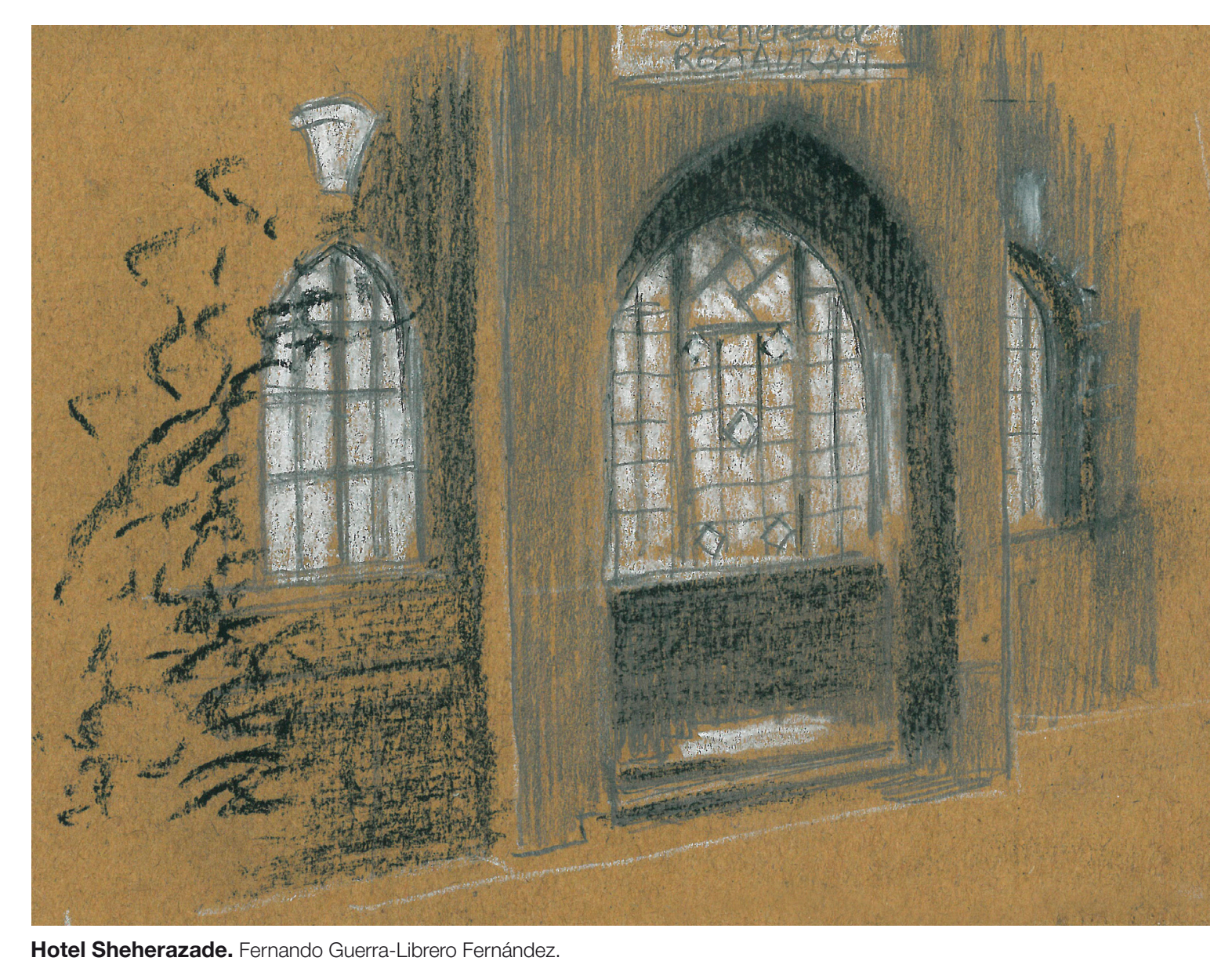




\section{Índice | Contents}

Trabajos de Egiptología está producida por

de la Universidad de La Laguna

$\mathrm{C} /$ Blanco $1,2^{\circ}$

38400 Puerto de la Cruz

España

( De los textos: sus autores y Trabajos de Egiptología

Diseño de arte y maquetación

Amparo Errandonea

Imprime: Gráfica Los Majuelos

Depósito Legal: TF 935-2015
José Miguel BARRIOS MUFREGE Covadonga en la Caldera del Teide 5

Foreword

Carta a una hermana en la luz / Letter to an enlightened sister 11

Estudio preliminar de la cerámica procedente de las excavaciones en la $\Pi \pi$ 209, Luxor,

Egipto

ZUlema BARAHONA MENDIETA

Amparo ERRANDONEA Liebestod 31

La ocupación macedónica y la Dinastía Lágida. Impacto político, económico y social Francisco BOSCH PUCHE

$$
\text { Cristina GIL PANEQUE Et il pleut sur Bruxelles } 75
$$

Reflexiones sobre Meretseguer en la estela EA272 del British Museum

Elisa CASTEL RONDA

Fernando GUERRA-LIBRERO FERNÁNDEZ Taftich 93

Ramesses III as Guarantor of Maat: the Iconographic Evidence at Medinet Habu Salvador COSTA, Teresa MAGADÁN

Fernando GUERRA-LIBRERO FERNÁNDEZ Muro de la casa de excavación 115

Iconographic Rendering of the Notion of Purification in Two Elements Included in the Vignettes of Chapters 17 and 125 of the Book of the Dead

ucía DÍAZ-IGLESIAS LLANOS

$$
\text { Isabel SÁNCHEZ MARQUÉS Adoratriz del Dios } 163
$$

Taboo - bwt?

Paul John FRANDSEN

Fernando GUERRA-LIBRERO FERNÁNDEZ Barco en el Nilo 193

Flat-Section Hairpins during the Egyptian Predynastic Period? A Proposa

$$
\text { of Identification and Typology }
$$

Candelaria MARTÍN DEL RÍO ÁLVAREZ

Isabel SÁNCHEZ MARQUÉS Plañidera 211 
José Miguel BARRIOS MUFREGE Covandonga en el patio de la TT 209, excavando el depósito de momificación 243

\section{The Courtyard of TT 209 (Areas C1 and C2). Seasons 2012 to 2014}

Miguel Ángel MOLINERO POLO, Cristo Manuel HERNÁNDEZ GÓMEZ

Hassan MOHAMED ALI, Saad BAKHIT ABD EL HAFEZ

Daniel Miguel MÉNDEZ RODRíGUEZ, Fernando GUERRA-LIBRERO FERNÁNDEZ,

Carlos GARCÍA ÁVILA, Lucía DíAZ-IGLESIAS LLANOS,

Zulema BARAHONA MENDIETA, José Miguel BARRIOS MUFREGE,

Paloma COLL TABANERA

Isabel SÁNCHEZ MARQUÉS Papiros 271

Algunas reflexiones sobre Uluburun y el intercambio comercial y cultural

en el Mediterráneo Oriental

Antonio PÉREZ LARGACHA

Fernando GUERRA-LIBRERO FERNÁNDEZ Hotel Sheherazade 289

The Framework of the Meeting. Narrative Uses of Stelae in Egyptian Literary Texts José Ramón PÉREZ-ACCINO

Sacha GÓMEZ MOÑIVAS En recuerdo de Covadonga 301

\section{Introducción a la investigación arqueológica para estudiantes}

a través de reconstrucciones virtuales

Sofía PÉREZ-RUIZ, Ainara ACEBO, Pilar RODRÍGUEZ MARÍN, Sacha GÓMEZ MOÑIVAS

Isabel SÁNCHEZ MARQUÉS Out of the blue 311

Lucernas decoradas con la imagen de una rana del yacimiento de Oxirrinco,

El-Bahnasa, Egipto

Esther PONS MELLADO

Submission guidelines
245

A Covadonga le gustaba conducir, le apasionaba estar al volante de su coche. "Pareces una chica A del futurismo italiano", le decía un amigo, aunque su primer automóvil fuera pequeño, blanco con un techo amarillo y a veces le costase llegar a su destino. Estuvimos años riendo cuando nos acordábamos del nombre con que habíamos bautizado aquella máquina. En nuestro recuerdo, ella sigue siendo la joven radiante y activa que conocimos a sus veinte años. Y su personalidad permanece entre nosotros como un perfume indeleble, retomando los versos del poeta alejandrino que tanto le gustaba. Quienes asistieron a sus conferencias conocieron la calidez de su voz; sus clases en la universidad crearon vocaciones; son, sin embargo, los que compartieron con ella su amistad quienes disfrutaron de los rasgos más sobresalientes de su personalidad: la generosidad, la entrega desinteresada a los demás. Cova tenía una capacidad excepcional para la empatía hacia los que se le acercaban. Fso le hizo ganar afectos en todos los ámbitos de su vida y conservarlos, desde los compros des tributo proser. Este y la huella que dejó en su generación.

Un grupo de amigos que vivimos con ella sus labores de docencia, investigación o proyectos arqueológicos, decidimos rendirle un homenaje particular, uno más entre los que se le han dedicado desde el momento en que su $k a$ voló al cielo. Este volumen es el resultado de esa voluntad de crear nuestro monumento a su memoria, por tantas experiencias inolvidables compartidas. El homenaje ha querido ser un caleidoscopio de miradas desde las que reflejar la personalidad de Covadonga y hemos preferido romper el formato académico tradicional. Los artículos se entrelazan con fotografías, dibujos, semblanzas o poemas que pretenden dejar constancia de la huella que ella legó a sus autores. Es nuestra ofrenda para que su $b a$ siga regresando desde donde esté hasta nosotros, cada vez que la nombremos y en cada ocasión en que su recuerdo tome forma en nuestro corazón. 


\section{Foreword}

Covadonga enjoyed driving; she loved being behind the wheel of her car. A Uriend used to tell her "you look like a woman of the Italian Futurism," although her first car was small and white with a yellow roof, and sometimes had difficulties reaching its destination. We laughed for years remembering the name with which we baptized that machine. In our memories, she is still the radiant and active young woman we met in her twenties, and her personality remains among us as an indelible scent, to draw upon the verse of an Alexandrian poet that she loved so much. Those who attended her lectures knew the warmth of her voice, her classes at the university created vocations, however, it is those who shared her friendship who enjoyed the most outstanding features of her personality: generosity, and selfless dedication to others. Cova had an exceptional capacity for empathy toward those who approached her This allowed her to win affection in all aspects of herfe and to retain it, from the classmates of her early schooldays, to the a circles of all a the generations as the legacy of an exceptional individual, and the mark she left on her generation.

As a group of friends who lived with her through teaching, research, or archaeological projects, we have decided to pay her a particular tribute; one more among the many that have been dedicated to her from the moment her ka flew to heaven. This volume is the result of our desire to create for her a monument to so many unforgettable shared experiences. We decided that this tribute should be a kaleidoscope, to reflect Covadonga's personality, and we have thus preferred to break from the traditional academic format. The articles are intertwined with photographs, drawings, sketches, or poems that are intended to record the traces she left with their authors. It is our offering so that her ba keeps coming back to us from where she now is, every time that her memory takes shape in our hearts.

\section{Carta a una hermana en la luz} SON le habla a su padre, como la hija que le habla a su madre.

¡O Senet, Senet Meret! Que Osiris-Khentamentiu te otorgue millones de años respirando aliento en tu nariz, dándote pan y cerveza junto a Hathor, Señora de la Tierra de Luz.

Tu condición es como la vida millones de veces, por orden de los dioses que están en el cielo y la en tierra. Que Ha, Señor de Occidente, actúe en tu favor de acuerdo a sus deseos, que Anubis, Señor del Buen Entierro actúe para ti como él lo quiera. Que pueda levantar una barrera contra los enemigos, hombres y mujeres malvados que se oponen a tu casa, tus hermanos, tus padres, tu memoria y tus hombres
obras.

Fuiste excelente en la tierra, por lo que también serás capaz y eficiente en el Más Allá. Que se te hagan ofrendas, que se realice la fiesta Haker para ti, que hagan la fiesta del Wag, que te den pan y cerveza del altar de Khentamentiu. Que puedas viajar río abajo en la Barca del Ocaso y que navegues río arriba en la Barca de la Mañana. Que estés justificada junto a cada dios. Que te conviertas en río arriba en la Barca de la Mañana. Que estés justificada junto a
alguien elogiado por nuestros espíritus masculinos y femeninos.

¿Has visto estos lamentos ahora que estás allí en el Más Allá?

Oh, gran dolor! Útil es una queja para hablar de lo que se hace contra nosotros de una manera tan injusta. Aunque no hay nada que hayamos hecho contra los dioses, y aunque no hemos comido de sus ofrendas, jsin embargo nos han privado de ti

Te han traído aquí a la Ciudad de la Eternidad, sin que albergues ira contra nosotros. Pero si hubiera un reproche en tu corazón, olvídalo por el bien de tus hermanas y hermanos. Sé misericordiosa, sé misericordiosa, y así todos los dioses del nomo de This serán misericordiosos contigo.

Mantén alejadas todas las aflicciones dirigidas a nosotros, tus hermanas y hermanos, porque tú sabes que tenemos una gran necesidad de esto. Que vivas para nosotros y así el Grande te elogie. Que la cara del gran dios sea gozosa para ti, y que él te dé pan puro con ambas manos.

Todos los sacrificios funerarios se han realizado para la que está en la luz, a fin de que pueda interceder por nosotros, los sobrevivientes en la tierra que han quedado atrás. Por lo tanto, busca que el que causó aquello de lo que estamos sufriendo ahora te dé una explicación, porque necesitamos entender y queremos también ser justificados delante de los dioses como lo eres ahora, entendiendo todo, justificada y transfigurada.

Son los hermanos y hermanas quienes le hablan a su hermana, para quien la luz ya nunca se oscurecerá. 


\section{Letter to an enlightened sister}

is the brothers and sisters who are speaking to their sister like the son who is speaking to
his father, like the daughter who is speaking to her mother.

O Senet, Senet Meret! May Osiris-Khentamentiu make millions of years for you by breathing breath into your nose, by giving bread and beer beside Hathor, Lady of the Land-of-Light.

Your condition is like life millions of time, by command of the gods who are in heaven and earth May $\mathrm{Ha}$, Lord of the West, act on your behalf as he wishes, may Anubis, Lord of the Good Burial act for you as he wishes. May you erect a barrier against male and female enemies, male and female evil ones who oppose your house, your brother, your mother, your memory, your deeds.

You are one who was excellent on earth, therefore you will also be capable in the hereafter. May one make offerings to you, may one make the Haker-feast for you, may one make the Wag-feast for you, may one give you bread and beer from the altar of Khentamentiu. You will travel downstream in the Bark-of-the-Evening and sail upstream in the Bark-of-the-Morning. May you be given justification at the side of every god. Make yourself into someone praised by our male and female ghosts.

Have you seen this lamentation now that you are there in the hereafter?

$\mathrm{O}$, great grief! Useful is a complaint to speaking concerning this which is done against us in such an unjust way, although there is nothing that we have done against the gods, and although we have not eaten of his offerings, nevertheless they have deprived us of you!

You have been brought here to the City of Eternity, without you harbouring anger against us. But if there is a reproach in your heart, forget it for the sake of your sisters and brothers. Be merciful, be merciful, then all the gods of the Thinite nome will be merciful towards you.

Keep away all afflictions directed at us, your sisters and brothers, for you know we have a need for this. May you live for us in order for the Great One to praise you. May the face of the great god be joyous because of you, so that he will give you pure bread with both his hands.

All mortuary sacrifices are made for the enlightened one in order to intercede on behalf of the inhabitants of earth. Therefore seek an explanation from him who caused that of which we are suffering now, for we want to be justified in front of the gods same as you are now.

It is the brothers and sisters who are speaking to their sister, she for whom the light will never darken. 